\title{
RANGES OF SYLVESTER MAPS AND A MINIMAL RANK PROBLEM*
}

\author{
ANDRE C.M. RAN ${ }^{\dagger}$ AND LEIBA RODMAN $\ddagger$
}

\begin{abstract}
It is proved that the range of a Sylvester map defined by two matrices of sizes $p \times p$ and $q \times q$, respectively, plus matrices whose ranks are bounded above, cover all $p \times q$ matrices. The best possible upper bound on the ranks is found in many cases. An application is made to a minimal rank problem that is motivated by the theory of minimal factorizations of rational matrix functions.
\end{abstract}

Key words. Sylvester maps, Invariant subspaces, Rank.

AMS subject classifications. 15A06, 15A99.

1. Introduction. Let $\mathbb{F}$ be a (commutative) field. We let $\mathbb{F}^{p \times q}$ stand for the set of $p \times q$ matrices with entries in $\mathbb{F} ; \mathbb{F}^{p \times 1}$ will be abbreviated to $\mathbb{F}^{p}$. The following minimal rank problem was stated in $[8$, Section 6$]$ for the case when $\mathbb{F}$ is the complex field $\mathbb{C}$ :

Problem 1.1. Given $A \in F^{n \times n}$, and given an $A$-invariant subspace $\mathcal{M} \subseteq \mathbb{F}^{n}$, find the smallest possible rank, call it $\mu(A, \mathcal{M})$, for the difference $A-Z$, where $Z$ runs over the set of all $n \times n$ matrices with entries in $\mathbb{F}$ for which there is a $Z$ invariant subspace $\mathcal{N} \subseteq \mathbb{F}^{n}$ complementary to $\mathcal{M}$. Also, find structural properties, or description, of such matrices $Z$.

The problem (for $\mathbb{F}=\mathbb{C}$ ) is intimately connected with minimal factorizations of rational matrix functions, in particular, if certain additional symmetry properties of $A, \mathcal{M}$, and $Z$ are assumed; see [8] for more details. Pairs of matrices $(A, Z)$ that have a pair of complementary subspaces $\mathcal{M}, \mathcal{N}$, of which the first is $A$-invariant and the second is $Z$-invariant, but without explicit rank conditions on $A-Z$, are studied in $[1,2]$, for example, in connection with complete minimal factorization of rational matrix functions.

${ }^{*}$ Received by the editors October 12, 2009. Accepted for publication January 20, 2010. Handling Editor: Harm Bart.

${ }^{\dagger}$ Afdeling Wiskunde, Faculteit der Exacte Wetenschappen, Vrije Universiteit Amsterdam, De Boelelaan 1081a, 1081 HV Amsterdam, The Netherlands (ran@cs.vu.nl).

${ }^{\ddagger}$ College of William and Mary, Department of Mathematics, P.O.Box 8795, Williamsburg, VA 23187-8795, USA (lxrodm@math.wm.edu). The research of this author was partially supported by the European Network for Analysis and Operator Theory, and by College of William and Mary Faculty Research Assignment and Plumeri Award. The work was completed during this author's visit at the Afdeling Wiskunde, Vrije Universiteit, whose hospitality is gratefully acknowledged. 
In the general formulation, Problem 1.1 appears to be difficult, even intractable, especially the part concerning properties or description of all matrices $Z$. To illustrate, assume $\mathbb{F}$ is algebraically closed, and let

$$
A=\left(\begin{array}{ll}
0 & 1 \\
0 & 0
\end{array}\right), \quad \mathcal{M}=\operatorname{Span}\left(\begin{array}{l}
1 \\
0
\end{array}\right) .
$$

Then $\mu(A, \mathcal{M})=1$, and a matrix $Z=\left(\begin{array}{ll}x & w \\ y & z\end{array}\right), x, y, z, w \in \mathbb{F}$, has the properties that $\operatorname{rank}(A-Z)=1$ and $Z$ has an invariant subspace complemented to $\mathcal{M}$ if and only if $Z$ is not a nonzero scalar multiple of $A$ and the equality $x z+y(1-w)=0$ holds.

If $A$ and $\mathcal{M}$ are as in Problem 1.1, by applying a similarity transformation we can assume without loss of generality that $\mathcal{M}$ is spanned by first $p$ unit coordinate vectors in $\mathbb{F}^{n}$; thus $A$ has the block form

$$
A=\left(\begin{array}{cc}
A_{1} & A_{12} \\
0 & A_{2}
\end{array}\right),
$$

where $A_{1} \in \mathbb{F}^{p \times p}, A_{2} \in \mathbb{F}^{(n-p) \times(n-p)}$. If the minimal polynomials of $A_{1}$ and $A_{2}$ are coprime, then it is easy to see that $\mu(A, \mathcal{M})=0$, i.e., $A$ has an invariant subspace $\mathcal{N}$ complementary to $\mathcal{M}$. Indeed, such $\mathcal{N}$ is spanned by the columns of $\left(\begin{array}{c}Q \\ I\end{array}\right)$, where the matrix $Q$ satisfies the equation

$$
\left(\begin{array}{cc}
A_{1} & A_{12} \\
0 & A_{2}
\end{array}\right)\left(\begin{array}{c}
Q \\
I
\end{array}\right)=\left(\begin{array}{c}
Q \\
I
\end{array}\right) A_{2}
$$

or

$$
Q A_{2}-A_{1} Q=A_{12}
$$

It is well known that the Sylvester map $Q \mapsto Q A_{2}-A_{1} Q$ is invertible if and only if the minimal polynomials of $A_{1}$ and $A_{2}$ are coprime. See, e.g., $[5,7]$ for this fact; although this was established in [5, 7] only for the complex field, the extension to any algebraically closed field is immediate, and to prove this fact for the general field $\mathbb{F}$ one considers the algebraic closure of $\mathbb{F}$. Hence (1.1) can be solved for $Q$ for any given $A_{12}$, and $\mu(A, \mathcal{M})=0$ is established. This example shows close connections of Problem 1.1 with properties of Sylvester maps. There is a large literature (in mathematical and engineering journals) on numerical analysis involving Sylvester maps; see, e.g., $[3,4]$ and the references cited there.

Connected to the Sylvester map the following problem appears in the theory of control for coordination (see [6]). Given is a linear system $\dot{x}(t)=A x(t)$, where the 
matrix $A$ has the form

$$
A=\left(\begin{array}{ccc}
A_{11} & 0 & A_{1 c} \\
0 & A_{22} & A_{2 c} \\
0 & 0 & A_{c c}
\end{array}\right)
$$

with respect to a fixed decomposition $X=X_{1} \dot{+} X_{2} \dot{+} X_{c}$ of the state space. One allows transformations $A \mapsto S^{-1} A S$, where $S$ is of the form

$$
S=\left(\begin{array}{ccc}
I & 0 & S_{1} \\
0 & I & S_{2} \\
0 & 0 & I
\end{array}\right)
$$

Then

$$
S^{-1} A S=\left(\begin{array}{ccc}
A_{11} & 0 & A_{11} S_{1}-S_{1} A_{c c}+A_{1 c} \\
0 & A_{22} & A_{22} S_{2}-S_{2} A_{c c}+A_{2 c} \\
0 & 0 & A_{c c}
\end{array}\right) .
$$

¿From the point of view of communicating as little as possible between the coordinator acting in $X_{c}$ and the subsystems acting in $X_{1}$ and $X_{2}$, it is of interest to study when the ranks of $A_{i i} S_{i}-S_{i} A_{c c}+A_{i c}$ are as small as possible for $i=1,2$. It is precisely this problem we shall discuss in the next section.

2. Ranges of Sylvester maps. We recall the definition of invariant polynomials. For $A \in \mathbb{F}^{p \times p}$, we let

$$
\lambda I-A=E(\lambda) \operatorname{diag}\left(\phi_{A, 1}(\lambda), \ldots, \phi_{A, p}(\lambda)\right) F(\lambda)
$$

where $E(\lambda), F(\lambda)$ are everywhere invertible matrix polynomials, and $\phi_{A, j}$ are scalar monic polynomials such that $\phi_{A, j}$ is divisible by $\phi_{A, j+1}$, for $j=1, \ldots, p-1$. The polynomials $\phi_{A, j}$ are called the invariant polynomials of $A ; \phi_{A, 1}$ is in fact the minimal polynomial of $A$.

For two matrices $A_{1}$ and $A_{2}$ over the field $\mathbb{F}$ of sizes $p \times p$ and $q \times q$, respectively, define the nonnegative integer $s\left(A_{1}, A_{2}\right)$ as

$$
\max \left\{j \mid 1 \leq j \leq \min \{p, q\}, \phi_{A_{1}, j}(\lambda) \text { and } \phi_{A_{2}, j}(\lambda) \text { are not coprime }\right\} \text {. }
$$

The maximum of the empty set in this formula is assumed to be zero. Clearly, $s\left(A_{1}, A_{2}\right)=0$ if and only if the minimal polynomials of $A_{1}$ and $A_{2}$ are coprime.

If all eigenvalues of $A_{1}$ and $A_{2}$ are in $\mathbb{F}$ (in particular if $\mathbb{F}$ is algebraically closed), then

$$
s\left(A_{1}, A_{2}\right)=\max _{\lambda \in \mathbb{F}} \min \left\{\operatorname{dim} \operatorname{Ker}\left(A_{1}-\lambda I\right), \operatorname{dim} \operatorname{Ker}\left(A_{2}-\lambda I\right)\right\} .
$$


Consider the linear Sylvester map (for fixed $A_{1}$ and $A_{2}$ ) $T: \mathbb{F}^{p \times q} \longrightarrow \mathbb{F}^{p \times q}$ defined by

$$
T(S)=S A_{2}-A_{1} S, \quad S \in \mathbb{F}^{p \times q},
$$

THEOREM 2.1.

(a) Every matrix $X \in \mathbb{F}^{p \times q}$ can be written in the form

$$
X=T(S)+Y,
$$

for some $S \in \mathbb{F}^{p \times q}$ and some $Y \in \mathbb{F}^{p \times q}$ with $\operatorname{rank} Y \leq s\left(A_{1}, A_{2}\right)$.

(b) Assume that $s\left(A_{1}, A_{2}\right) \neq 0$, and that the greatest common divisor of the minimal polynomials of $A_{1}$ and $A_{2}$ have all their roots in $\mathbb{F}$ (in particular, this condition is always satisfied if $\mathbb{F}$ is algebraically closed). Then for fixed $A_{1}$ and $A_{2}$, there is a Zariski open nonempty set $\Omega$ of $\mathbb{F}^{p \times q}$ such that for every $X \in \Omega$, there is no representation of $X$ in the form

$$
X=T(S)+Y,
$$

where $S, Y \in \mathbb{F}^{p \times q}$ are such that $\operatorname{rank} Y<s\left(A_{1}, A_{2}\right)$.

Theorem 2.1 can be thought of as a generalization of the well known fact that the Sylvester map is a bijection if and only if the minimal polynomials of $A_{1}$ and $A_{2}$ are coprime.

Consider the following example to illustrate Theorem 2.1. Let

$$
A_{1}=\left[\begin{array}{lll}
0 & 0 & 0 \\
0 & 0 & 0 \\
0 & 0 & 1
\end{array}\right] \text { and } A_{2}=\left[\begin{array}{ccc}
0 & 0 & 0 \\
0 & 1 & 0 \\
0 & 0 & 1
\end{array}\right] \text {. }
$$

The invariant polynomials are

$$
\begin{gathered}
\phi_{A_{1}, 1}(\lambda)=\phi_{A_{2}, 1}(\lambda)=\lambda(\lambda-1) \\
\phi_{A_{1}, 2}(\lambda)=\lambda, \quad \phi_{A_{1}, 2}(\lambda)=\lambda-1, \quad \phi_{A_{1}, 3}(\lambda)=\phi_{A_{2}, 3}(\lambda)=1 .
\end{gathered}
$$

We have $s\left(A_{1}, A_{2}\right)=1$. The range of the Sylvester map is easy to find:

$$
\text { Range } T=\left\{\left[\begin{array}{ccc}
0 & * & * \\
0 & * & * \\
* & 0 & 0
\end{array}\right]\right\}
$$


where by ${ }^{*}$ we denote arbitrary entries which are independent free variables. For every $X=\left[x_{i, j}\right] \in \mathbb{F}^{3 \times 3}$, we have

$$
X=\left[\begin{array}{c}
x_{1,1} \\
x_{2,1} \\
1
\end{array}\right]\left[\begin{array}{lll}
1 & x_{3,2} & x_{3,3}
\end{array}\right]+\left[\begin{array}{ccc}
0 & * & * \\
0 & * & * \\
* & 0 & 0
\end{array}\right]
$$

3. Proof of Theorem 2.1. Part (a). We use the rational canonical forms for $A_{1}$ and $A_{2}$ (see, e.g., [5]), together with the invariance of the statement of the theorem and of its conclusions under similarity transformations

$$
A_{1} \longrightarrow G_{1}^{-1} A_{1} G_{1}, \quad A_{2} \longrightarrow G_{2}^{-1} A_{2} G_{2}, \quad G_{1} \text { and } G_{2} \text { invertible. }
$$

We may assume therefore without loss of generality that

$$
A_{1}=\operatorname{diag}\left(A_{1}^{(1)}, \ldots, A_{1}^{(u)}\right) \quad \text { and } \quad A_{2}=\operatorname{diag}\left(A_{2}^{(1)}, \ldots, A_{2}^{(v)}\right),
$$

where

$$
A_{1}^{(j)}=\operatorname{diag}\left(A_{1}^{(j, 1)}, \ldots, A_{1}^{\left(j, \gamma_{1, j}\right)}\right), \quad j=1, \ldots, u,
$$

and where the characteristic polynomials of $A_{1}^{(j, k)}, k=1, \ldots, \gamma_{1, j}$, are all powers of the same monic irreducible polynomial $f_{1, j}$. The matrices $A_{1}^{(j, k)}$ are nonderogatory, i.e., the minimal and the characteristic polynomials of $A_{1}^{(j, k)}$ coincide. In addition, we require that the irreducible polynomials $f_{1,1}, \ldots, f_{1, u}$ are all distinct.

Similarly,

$$
A_{2}^{(j)}=\operatorname{diag}\left(A_{2}^{(j, 1)}, \ldots, A_{2}^{\left(j, \gamma_{2, j}\right)}\right), \quad j=1, \ldots, v
$$

where the characteristic polynomials of $A_{2}^{(j, k)}, k=1, \ldots, \gamma_{2, j}$, are all powers of the same monic irreducible polynomial $f_{2, j}$, and the polynomials $f_{2,1}, \ldots, f_{2, v}$ are all distinct. Again, the matrices $A_{2}^{(j, k)}$ are nonderogatory.

Moreover, we arrange the blocks $A_{1}^{(1)}, \ldots, A_{1}^{(u)}$ and $A_{2}^{(1)}, \ldots, A_{2}^{(v)}$ so that

$$
f_{1,1}=f_{2,1}, \ldots, f_{1, \ell}=f_{2, \ell},
$$

but the irreducible polynomials

$$
f_{1,1}, \ldots, f_{1, u}, f_{2, \ell+1}, \ldots, f_{2, v}
$$

are all distinct. (The case when $\ell=0$, i.e., the polynomials $f_{1, j}$ and $f_{2, j}$ are all distinct, is not excluded; in this case the subsequent arguments should be modified in 
obvious ways.) Note that since powers of distinct irreducible polynomials are coprime, it follows that the characteristic polynomials of the matrices

$$
A_{1}^{(1)}, \ldots, A_{1}^{(u)}, A_{2}^{(\ell+1)}, \ldots, A_{2}^{(v)}
$$

are pairwise coprime.

We assume in addition that $A_{1}^{(j, k)}$ are companion matrices. To set up notation, we let $e_{j}$ be a row with 1 in the $j$ th position and zeros in all other positions (the number of components in $e_{j}$ will be evident from context), and analogously let $e_{j}^{T}$ (the transpose of $e_{j}$ ) be the column with 1 in the $j$ th position and zeros in all other positions. Let $\xi_{1, j, k}$ (resp., $\xi_{2, j, k}$ ) be the size of the matrix $A_{1}^{(j, k)}$ (resp., $A_{2}^{(j, k)}$ ). Thus, we let

$$
A_{1}^{(j, k)}=\left[\begin{array}{c}
e_{2} \\
e_{3} \\
\vdots \\
e_{\xi_{1, j, k}} \\
\alpha_{1, j, k}
\end{array}\right]
$$

or

$$
A_{1}^{(j, k)}=\left[\begin{array}{lllll}
e_{2}^{T} & e_{3}^{T} & \ldots & e_{\xi_{1, j, k}}^{T} & \alpha_{1, j, k}^{T}
\end{array}\right]
$$

for some row $\alpha_{1, j, k}$ (with entries in $\mathbb{F}$ ), and analogously,

$$
A_{2}^{(j, k)}=\left[\begin{array}{c}
e_{2} \\
e_{3} \\
\vdots \\
e_{\xi_{2, j, k}} \\
\alpha_{2, j, k}
\end{array}\right]
$$

or

$$
A_{2}^{(j, k)}=\left[\begin{array}{lllll}
e_{2}^{T} & e_{3}^{T} & \ldots & e_{\xi_{2, j, k}}^{T} & \alpha_{2, j, k}^{T}
\end{array}\right]
$$

for some row $\alpha_{2, j, k}$.

The forms (3.3) and (3.5) will be used if $\gamma_{1, j} \leq \gamma_{2, j}$, and the forms (3.4) and (3.6) will be used if $\gamma_{1, j}>\gamma_{2, j}$.

We return to the Sylvester map T. Conformably with (3.1), we partition

$$
S=\left[S_{j_{1}, j_{2}}\right]_{j_{1}=1, \ldots, u ; j_{2}=1, \ldots, v} .
$$


Thus,

$$
T(S)=S A_{2}-A_{1} S=\left[S_{j_{1}, j_{2}} A_{2}^{\left(j_{2}\right)}-A_{1}^{\left(j_{1}\right)} S_{j_{1}, j_{2}}\right]_{j_{1}=1, \ldots, u ; j_{2}=1, \ldots, v} .
$$

Also, if $X \in \mathbb{F}_{p \times q}$ is an arbitrary matrix, then we partition again conformably with $(3.1)$ :

$$
X=\left[X_{j_{1}, j_{2}}\right]_{j_{1}=1, \ldots, u ; j_{2}=1, \ldots, v}
$$

We will show that for any given $X \in \mathbb{F}^{p \times q}$, there exist

$$
S_{j, j} \in \mathbb{F}^{\left(\xi_{1, j, 1}+\cdots+\xi_{1, j, \gamma_{1, j}}\right) \times\left(\xi_{2, j, 1}+\cdots+\xi_{2, j, \gamma_{2, j}}\right)}, \quad j=1, \ldots, \ell,
$$

with the property that

$$
X_{j, j}=Y_{j, j}+\left(S_{j, j} A_{2}^{(j)}-A_{1}^{(j)} S_{j, j}\right)
$$

for some matrix $Y_{j, j}$ such that

$$
\operatorname{rank} Y_{j, j} \leq \min \left\{\gamma_{1, j}, \gamma_{2, j}\right\}, \quad j=1, \ldots, \ell .
$$

Assuming that we have already shown the existence of $S_{j, j}$ satisfying (3.8) and (3.7), we can easily complete the proof of Part (a).

Indeed, let

$$
\mu=\max _{j=1, \ldots, \ell}\left(\min \left\{\gamma_{1, j}, \gamma_{2, j}\right\}\right)
$$

and notice that $\mu=s\left(A_{1}, A_{2}\right)$. Now let

$$
Y_{j, j}=W_{j, j} Z_{j, j}, \quad j=1, \ldots, \ell
$$

be a rank decomposition, where the matrix $W_{j, j}$ (resp., $Z_{j, j}$ ) has $\mu$ columns (resp., $\mu$ rows). We also put formally $W_{j, j}=0$ for $j=\ell+1, \ldots, u$, and and $Z_{j, j}=0$ for $j=\ell+1, \ldots, v$. Using the property that the characteristic polynomials of matrices (3.2) are pairwise coprime, and that the Sylvester map $S \mapsto S B_{2}-B_{1} S$ is onto if the characteristic polynomials of $B_{1}$ and $B_{2}$ are coprime, we find

$$
S_{j_{1}, j_{2}} \in \mathbb{F}^{\left(\xi_{1, j_{1}, 1}+\cdots+\xi_{1, j_{1}, \gamma_{1, j_{1}}}\right) \times\left(\xi_{2, j_{2}, 1}+\cdots+\xi_{2, j_{2}, \gamma_{2, j_{2}}}\right)}
$$

for

$$
j_{1}=1, \ldots, u, \quad j_{2}=1, \ldots, v, \quad\left(j_{1}, j_{2}\right) \notin\{(1,1) \ldots,(\ell, \ell)\},
$$

such that

$$
X_{j_{1}, j_{2}}=W_{j_{1}, j_{1}} Z_{j_{2}, j_{2}}+\left(S_{j_{1}, j_{2}} A_{2}^{\left(j_{2}\right)}-A_{1}^{\left(j_{1}\right)} S_{j_{1}, j_{2}}\right)
$$


Now letting

$$
Y=\left[\begin{array}{c}
W_{1,1} \\
W_{2,2} \\
\vdots \\
W_{u, u}
\end{array}\right]\left[\begin{array}{llll}
Z_{1,1} & Z_{2,2} & \cdots & Z_{v, v}
\end{array}\right]
$$

we have $X=T(S)+Y$, and obviously, $\operatorname{rank} Y \leq \mu$.

Thus, it remains to show the existence of $S_{j, j}$ satisfying (3.7) and (3.8). We fix $j, j=1, \ldots, \ell$. We assume that $\gamma_{1, j} \leq \gamma_{2, j}$, thus (3.3) and (3.5) will be used; if $\gamma_{1, j}>\gamma_{2, j}$ the proof is completely analogous using (3.4) and (3.6). Choose rows $\alpha_{1, j, k}^{\prime}$ $\left(k=1, \ldots, \gamma_{1, j}\right)$ so that the characteristic polynomials of the matrices

$$
B_{1}^{(j, k)}:=\left[\begin{array}{c}
e_{2} \\
e_{3} \\
\vdots \\
e_{\xi_{1, j, k}} \\
\alpha_{1, j, k}^{\prime}
\end{array}\right], \quad k=1, \ldots, \gamma_{1, j}
$$

are coprime to $f_{2, j}=f_{1, j}$, and let

$$
B_{1}^{(j)}=\operatorname{diag}\left(B_{1}^{(j, 1)}, \ldots, B_{1}^{\left(j, \gamma_{1, j}\right)}\right) .
$$

Therefore, we can find $S_{j, j}$ so that

$$
X_{j, j}=S_{j, j} A_{2}^{(j)}-B_{1}^{(j)} S_{j, j}
$$

Now (3.7) holds with

$$
Y_{j, j}=\left(B_{1}^{(j)}-A_{1}^{(j)}\right) S_{j, j}
$$

and since the matrix $Y_{j, j}$ has at most $\gamma_{1, j}$ nonzero rows, we have rank $Y_{j, j} \leq \gamma_{1, j}$, as required.

Part (b). We assume that $A_{1}$ and $A_{2}$ have the form (3.1), and use the notation introduced in the proof of Part (a). We have $\ell \geq 1$. Let $j_{0}$ be such that $\mu=$ $\min \left\{\gamma_{1, j_{0}}, \gamma_{2, j_{0}}\right\}$. Without loss of generality we may assume $j_{0}=1$. Let $p_{1} \times p_{1}$ and $q_{1} \times q_{1}$, be the size of

$$
A_{1}^{(1)}=\operatorname{diag}\left(A_{1}^{(1,1)}, \ldots, A_{1}^{\left(1, \gamma_{1,1}\right)}\right) \quad \text { and } \quad A_{2}^{(1)}=\operatorname{diag}\left(A_{2}^{(1,1)}, \ldots, A_{2}^{\left(1, \gamma_{2,1}\right)}\right),
$$

respectively. It is easy to see that it suffices to find a Zariski open nonempty set $\Omega_{1}$ of $\mathbb{F}^{p_{1} \times q_{1}}$ such that for every $X_{1} \in \Omega_{1}$, there is no representation of $X_{1}$ in the form $X_{1}=$ 
$\left(S A_{2}^{(1)}-A_{1}^{(1)} S\right)+Y$, where $S, Y \in F^{p_{1} \times q_{1}}$ and $\operatorname{rank} Y<\min \left\{\gamma_{1,1}, \gamma_{2,1}\right\}$. Because of the hypotheses of Part (b), we may assume that every matrix $A_{k}^{1, w}, w=1,2, \ldots, \gamma_{k, 1}$, $k=1,2$, is an (upper triangular) Jordan block with the same eigenvalue $\lambda$; let the size of this block be $p_{k, w} \times p_{k, w}$. Consider a matrix of the form $S A_{2}^{(1)}-A_{1}^{(1)} S, S \in \mathbb{F}^{p_{1} \times q_{1}}$, which is partitioned

$$
S A_{2}^{(1)}-A_{1}^{(1)} S=\left[Q_{\alpha, \beta}\right]_{\alpha=1, \beta=1}^{\gamma_{1,1}, \gamma_{2,1}},
$$

where the block $Q_{\alpha, \beta}$ has the size

$$
\left(\text { size of } A_{1}^{1, \alpha}\right) \times\left(\text { size of } A_{2}^{1, \beta}\right) .
$$

Since the $A_{k}^{1, w}$ 's are Jordan blocks, the bottom left corners of the blocks $Q_{\alpha, \beta}$ are all zeros. Now partition

$$
X_{1}=\left[X_{\alpha, \beta}\right]_{\alpha=1, \beta=1}^{\gamma_{1,1}, \gamma_{2,1}} \in \mathbb{F}^{p_{1} \times q_{1}}
$$

comformably with the right hand side of (3.9). The Zariski open set $\Omega_{1}$ consists of exactly those matriceds $X_{1}$ for which the $\gamma_{1,1} \times \gamma_{2,1}$ matrix formed by the bottom left corners of the $X_{\alpha, \beta}$ 's has the full rank, equal to $\min \left\{\gamma_{1,1}, \gamma_{2,1}\right\}$.

4. A special case of the minimal rank problem. Given a subspace $\mathcal{M} \subseteq \mathbb{F}^{n}$ and a matrix $Z \in \mathbb{F}^{n \times n}$, we say that $\mathcal{M}$ is a complementary $Z$-invariant subspace if $\mathcal{M}$ is $Z$-invariant and some direct complement to $\mathcal{M}$ in $\mathbb{F}^{n}$ is also $Z$-invariant. Denote by $\mathcal{C} \mathcal{I}(\mathcal{M})$ the set of all matrices $Z$ for which $\mathcal{M}$ is a complementary invariant subspace.

The following problem is closely related to Problem 1.1.

Problem 4.1. Given a matrix $A \in \mathbb{F}^{n \times n}$ and its invariant subspace $\mathcal{M} \subseteq \mathbb{F}^{n}$, find the smallest possible rank of the differences $A-Y$, where $Y$ is an arbitrary matrix in $\mathcal{C I}(\mathcal{M})$, and find a matrix $Z \in \mathcal{C} \mathcal{I}(\mathcal{M})$ such that the difference $A-Z$ has this smallest possible rank.

In fact, Problem 4.1 requires an extra condition for $Z$ in comparison with Problem 1.1 , namely, that $\mathcal{M}$ is an invariant subspace for $Z$.

Using similarity, we assume without loss of generality that

$$
\mathcal{M}=\left\{\left(\begin{array}{l}
x \\
0
\end{array}\right): x \in F^{p}\right\}, \quad A=\left(\begin{array}{cc}
A_{1} & A_{12} \\
0 & A_{2}
\end{array}\right), \quad A_{1} \in \mathbb{F}^{p \times p}, \quad A_{2} \in \mathbb{F}^{q \times q} .
$$

Theorem 2.1 sheds some light on Problem 4.1, as follows.

Theorem 4.2. Let $\kappa:=s\left(A_{1}, A_{2}\right)$. There exists a matrix $Z \in \mathcal{C} \mathcal{I}(\mathcal{M})$ such that

$$
\operatorname{rank}(A-Z) \leq \kappa .
$$


Moreover, $Z$ can be taken in the form

$$
Z=\left(\begin{array}{cc}
A_{1} & W \\
0 & A_{2}
\end{array}\right)
$$

for some $W$.

Proof. We use Theorem 2.1. Indeed, if $Z$ is in the form (4.2), then $Z \in \mathcal{C I}(\mathcal{M})$ if and only if for some matrix $Q \in \mathbb{F}^{p \times q}$ the subspace $\operatorname{Span}\left(\begin{array}{c}Q \\ I\end{array}\right)$ is $Z$-invariant, i.e., the equation

$$
\left(\begin{array}{cc}
A_{1} & W \\
0 & A_{2}
\end{array}\right)\left(\begin{array}{c}
Q \\
I
\end{array}\right)=\left(\begin{array}{c}
Q \\
I
\end{array}\right) A_{2}
$$

holds, or equivalently,

$$
Q A_{2}-A_{1} Q-\left(W-A_{12}\right)=A_{12}
$$

By Theorem 2.1, such $Q$ exists for some $W$ with the property that rank $\left(A_{12}-W\right) \leq \kappa$. Since obviously

$$
\operatorname{rank}(A-Z)=\operatorname{rank}\left(A_{12}-W\right)
$$

the result follows.

\section{REFERENCES}

[1] H. Bart and H. Hoogland. Complementary triangular forms of pairs of matrices, realizations with prescribed main matrices, and complete factorization of rational matrix functions. Linear Algebra Appl., 103:193-228, 1988.

[2] H. Bart and R.A. Zuidwijk. Simultaneous reduction to triangular forms after extension with zeroes. Linear Algebra Appl., 281:105-135, 1998.

[3] R. Bhatia and P. Rosenthal. How and why to solve the operator equation $A X-X B=Y$. Bull. London Math. Soc., 29(1):1-21, 1997.

[4] K. Datta. The matrix equation $X A-B X=R$ and its applications. Linear Algebra Appl., 109:91-105, 1988.

[5] F.R. Gantmacher. The Theory of Matrices. Chelsea Publishing Co., New York, 1959.

[6] P.L. Kempker, A.C.M. Ran, and J.H. van Schuppen. Construction of a coordinator for coordinated linear systems. Proceedings of the European Control Conference 2009, 2009.

[7] P. Lancaster and M. Tismenetsky. The Theory of Matrices With Applications, 2nd edition. Academic Press, Orlando, 1985.

[8] L. Lerer, M.A. Petersen, and A.C.M. Ran. Existence of minimal nonsquare $J$-symmetric factorizations for self-adjoint rational matrix functions. Linear Algebra Appl., 379:159-174, 2004. 